\title{
A VARIATION-OF-PARAMETERS INEQUALITY
}

\section{DAVID LOWELL LOVELADY}

ABSTRACT. Recent work in stability theory for linear ordinary differential equations has made much use of the inequalities which can be deduced from the variation-of-parameters formula. In this article it is shown that similar inequalities hold for nonlinear Stieltjes integral equations and hence, as a special case, for nonlinear differential equations in which the nonlinearity is of Lipschitz type.

I. Introduction. Recent work in stability theory for linear ordinary differential equations has made much use of the variationof-parameters formula (see, for example, Coppel [1, pp. 64-81] and Hallam [2], [3]). The applications of the formula are usually by way of inequalities which can be derived from it. In this article it will be shown that similar inequalities hold for nonlinear Stieltjes integral equations and hence, as a special case, for nonlinear differential equations in which the nonlinearity is of Lipschitz type.

II. Preliminaries. Let $X$ be a Banach space with norm $N_{1}$. Let $c$ be a real number, and let $S=[c, \infty)$. Let $H$ be the set to which $A$ belongs only in case $A$ is a function from $X$ to $X, A(0)=0$, and there is a number $b$ so that $N_{1}[A(p)-A(q)] \leqq b N_{1}[p-q]$ whenever each of $p$ and $q$ is in $X$. If $A$ is in $H$, let $N_{2}[A]$ be the least number $b$ so that $N_{1}[A(p)-A(q)] \leqq b N_{1}[p-q]$ whenever each of $p$ and $q$ is in $X$, and let $N_{3}[A]$ be the least number $b$ so that $N_{1}[A(p)] \leqq b N_{1}[p]$ whenever $p$ is in $X$. Let $B V H$ (respectively $B V X$ ) be the set to which $F$ belongs only in case $F$ is a function from $S$ to $H$ (respectively $X$ ) which is of bounded $N_{2}$-variation (respectively bounded $N_{1}$-variation) on each bounded interval of $S$. Let $O M$ be the set to which $W$ belongs only in case $W$ is an $H$-valued function on that subset of $S \times S$ to which $(t, s)$ belongs only in case $t \geqq s$, and

(i) $W(x, y) W(y, z)=W(x, z)$ whenever $x \geqq y \geqq z \geqq c$, where the multiplication is composition, and

(ii) if $a$ is in $S$, there is a number $b$ so that whenever $a=t_{0} \geqq t_{1}$ $\geqq \cdots \geqq t_{n} \geqq c$, then

$$
\sum_{k=1}^{n} N_{2}\left[W\left(t_{k-1}, t_{k}\right)-I\right] \leqq b,
$$

where $I$ in $H$ is given by $I[p]=p$.

Received by the editors March 2, 1970.

AMS 1969 subject classifications. Primary 3451; Secondary 3495.

Key words and phrases. Bounds, nonhomogeneous perturbations, stability, Stieltjes integral equations, variation-of-parameters. 
In [7], Mac Nerney showed that if $F$ is in $B V H$, there is exactly one member $W$ of $O M$ so that if $(s, p)$ is in $S \times X$, and $h$ is given by $h(t)=W(t, s)[p]$ for $t \geqq s, h(t)=0$ for $t<s$, then $h$ is in $B V X$ and if $t \geqq s$,

$$
h(t)=p+(L) \int_{s}^{t} d F[h],
$$

where the one term approximation for $(L) \int_{s}^{t} d F[h]$ is $F(t)[h(s)]$ $-F(s)[h(s)]$. If $F$ and $W$ are related as in the previous sentence, $W$ will be called the "fundamental solution for $F$ ".

Note that since $X$ is a Banach space, the set of real numbers can be considered as a subset of $H$. Let $B V R$ and $O M R$ consist of the realvalued members of $B V H$ and $O M$ respectively. Martin [9, Lemma 3.3, Theorem 3.1, Theorem 3.3] (see also [8, Theorem 1, Theorem 2]) has shown the following theorem.

THEOREM 1. Let $F$ be in BVH. If $(a, b)$ is in $S \times S$, then $\int_{a}^{b}\left(N_{z}[I+d F]-1\right)$ exists. If $\gamma$ is given on $S$ by $\gamma(t)=\int_{c}^{t}\left(N_{3}[I+d F]-1\right)$, then $\gamma$ is in $B V R$. If $W$ and $\lambda$ are fundamental solutions for $F$ and $\gamma$, respectively, then $N_{3}[W(s, t)] \leqq \lambda(s, t)$ whenever $s \geqq t$. Furthermore, $\lambda$ is the least member of $O M R$ for which this last inequality holds.

\section{The main theorems.}

Theorem A. Let $F, W, \gamma$, and $\lambda$ be as in Theorem 1 , and let $f$ be in $B V X$. Let $h$ be that member of $B V X$ such that if $t$ is in $S$, then

$$
h(t)=f(t)+(L) \int_{c}^{t} d F[h] .
$$

Then, if $t$ is in $S$,

$$
N_{1}[h(t)] \leqq \lambda(t, c) N_{1}[f(c)]+(R) \int_{c}^{t} \lambda(t, \quad) N_{1}[d f] .
$$

REMARK. Note that $G$, given by

$$
G(t)=\lambda(t, c) N_{1}[f(c)]+(R) \int_{c}^{t} \lambda(t,) N_{1}[d f],
$$

is that member of $B V R$ such that if $t$ is in $S$, then

$$
G(t)=N_{1}[f(c)]+\int_{c}^{t} N_{1}[d f]+(L) \int_{c}^{t}(d \gamma) G .
$$

(See Mac Nerney [6, Theorem 5.2].) 
Let $M$ be that real-valued function on $H$ given by

$$
M[A]=\lim _{t \rightarrow 0+}\left[(1 / t)\left(N_{3}[I+t A]-1\right)\right] .
$$

This is a straightforward generalization of the "measure" used in studying matrix differential equations (see, for example, Coppel [ 1 , p. 41]). Now we can get the following application of Theorem A to differential equations.

THEOREM B. Let $B$ be an $N_{2}$-continuous function from $S$ to $H$, and let $g$ be an $N_{1}$-continuous function from $S$ to $X$. Let $p$ be in $X$, and let $Y$ and $Z$ be continuously differentiable functions from $S$ to $X$ such that

(i) $Y(c)=Z(c)=p$,

(ii) $Y^{\prime}(t)=B(t)[Y(t)]$ if $t>c$, and

(iii) $Z^{\prime}(t)=B(t)[Z(t)]+g(t)$ if $t>c$.

Then, whenever $t$ is in $S$,

$$
\begin{aligned}
N_{1}[Z(t)] \leqq & N_{1}[p] \exp \left[\int_{c}^{t} M[B(r)] d r\right] \\
& +\int_{c}^{t} N_{1}[g(s)] \exp \left[\int_{s}^{t} M[B(r)] d r\right] d s .
\end{aligned}
$$

REMARK. Note that $G$, given by

$$
\begin{aligned}
G(t)= & N_{1}[p] \exp \left[\int_{c}^{t} M[B(r)] d r\right] \\
& +\int_{c}^{t} N_{1}[g(s)] \exp \left[\int_{c}^{t} M[B(r)] d r\right] d s,
\end{aligned}
$$

solves $G^{\prime}(t)=M[B(t)] G(t)+N_{1}[g(t)], G(c)=N_{1}[p]$. To see how Theorem $\mathrm{B}$ follows from Theorem A, let $F$ and $f$ be given by

$$
F(t)=\int_{c}^{t} B(r) d r \text { and } f(t)=P+\int_{0}^{t} g(r) d r .
$$

Martin [9, Example 3.1] (see also [8]) has shown that in this circumstance, $\gamma$ will be given by

$$
\gamma(t)=\int_{c}^{t} M[B(r)] d r .
$$

Before proving Theorem $\mathrm{A}$, we make a definition and cite a lemma.

Definition 1. A member $Q$ of $B V X$ shall be called a step function only in case there is an unbounded monotone sequence $\left(t_{k}\right)_{k=0}^{\infty}$ into $S$ 
with $c=t_{0}$, and two sequences $\left(a_{k}\right)_{k=0}^{\infty}$ and $\left(b_{k}\right)_{k=1}^{\infty}$ into $X$ so that if $k \geqq 0$, then $Q\left(t_{k}\right)=a_{k}$ and $Q(t)=b_{k+1}$ whenever $t_{k}<t<t_{k=1}$.

LEMma 1. If $f$ is in $B V X$, there is a $B V X$-valued sequence $\left(Q_{n}\right)_{n=1}^{\infty}$ such that

(i) for each $n, Q_{n}$ is a step function,

(ii) if $d$ is in $S$, $\sup _{n \geq 1} \int_{c}^{d} N_{1}\left[d Q_{n}\right] \leqq \int_{c}^{d} N_{1}[d f]$, and

(iii) $\lim _{n \rightarrow \infty} Q_{n}(t)=f(t)$ for each $t$ in $S$, the convergence being uniform on bounded subsets of $S$.

REMARK. Lemma 1 is an easy consequence of known results; see, for example, [5, Lemma 4.1b] and [4, Theorem 1.0].

In light of the lemma, it is clear that we only need prove Theorem $A$ in the case in which $f$ is a step function.

Proof of Theorem A. Suppose $f$ is a step function, and let $\left(t_{k}\right)_{k=0}^{\infty}$, $\left(a_{k}\right)_{k=0}^{\infty}$, and $\left(b_{k}\right)_{k=1}^{\infty}$ be the sequences describing $f$. Let $G$ be given by

Now, if $t$ is in $S$,

$$
G(t)=\lambda(t, c) N_{1}\left[a_{0}\right]+(R) \int_{c}^{t} \lambda(t,) N_{1}[d f] .
$$

$$
G(t)=N_{1}\left[a_{0}\right]+\int_{c}^{t} N_{1}[d f]+(L) \int_{c}^{t}(d \gamma) G .
$$

Clearly the conclusion holds if $t=c$. Now

so

$$
\begin{aligned}
h\left(c^{+}\right) & =b_{1}+\left[F\left(c^{+}\right)-F(c)\right][h(c)] \\
& =b_{1}+\left[F\left(c^{+}\right)-F(c)\right]\left[a_{0}\right] \\
& =\left(b_{1}-a_{0}\right)+\left[I+F\left(c^{+}\right)-F(c)\right]\left[a_{0}\right],
\end{aligned}
$$

$$
\begin{aligned}
N_{1}\left[h\left(c^{+}\right)\right] & \leqq N_{1}\left[b_{1}-a_{0}\right]+\left[1+\gamma\left(c^{+}\right)-\gamma(c)\right] N_{1}\left[a_{0}\right] \\
& =G\left(c^{+}\right) .
\end{aligned}
$$

So, if $t_{0}<t<t_{1}$,

$$
h(t)=h\left(c^{+}\right)+(L) \int_{c^{+}}^{t} d F[h], \quad h(t)=W\left(t, c^{+}\right)\left[h\left(c^{+}\right)\right],
$$

and

$$
N_{1}[h(t)] \leqq \lambda\left(t, c^{+}\right) N_{1}\left[h\left(c^{+}\right)\right] \leqq \lambda\left(t, c^{+}\right) G\left(c^{+}\right)=G(t) .
$$

Now

$$
\begin{aligned}
& h\left(t_{1}\right)=h\left(t_{1}^{-}\right)+a_{1}-b_{1}+\left[F\left(t_{1}\right)-F\left(t_{1}^{-}\right)\right]\left[h\left(t_{1}^{-}\right)\right], \\
& h\left(t_{1}\right)=a_{1}-b_{1}+\left[I+F\left(t_{1}\right)-F\left(t_{1}^{-}\right)\right]\left[h\left(t_{1}^{-}\right)\right],
\end{aligned}
$$


so

$$
\begin{aligned}
N_{1}\left[h\left(t_{1}\right)\right] & \leqq N_{1}\left[a_{1}-b_{1}\right]+\left[1+\gamma\left(t_{1}\right)-\gamma\left(t_{1}^{-}\right)\right] N_{1}\left[h\left(t_{1}^{-}\right)\right] \\
& \leqq N_{1}\left[a_{1}-b_{1}\right]+\left[1+\gamma\left(t_{1}\right)-\gamma\left(t_{1}^{-}\right)\right] G\left(t_{1}^{-}\right) \\
& =G\left(t_{1}\right) .
\end{aligned}
$$

By an obvious induction argument, the remainder of the proof is clear.

\section{REFERENCES}

1. W. A. Coppel, Stability and asymptotic behavior of differential equations, Heath, Boston, Mass., 1965. MR 32 \#7875.

2. T. G. Hallam, On the asymptotic growth of the solutions of a system of nonhomogeneous linear differential equations, J. Math. Anal. Appl. 25 (1969), 254-265. MR 38 \#2388.

3. - On asymptotic equivalence of the bounded solutions of two systems of differential equations, Michigan Math. J. 16 (1969), 353-363.

4. D. B. Hinton, $A$ Stieltjes-Volterra integral equation theory, Canad. J. Math. 18 (1966), 314-331. MR 32 \#6169.

5. R. E. Lane, The integral of a function with respect to a function, Proc. Amer. Math. Soc. 5 (1954), 59-66. MR 15, 514.

6. J.S. Mac Nerney, Integral equations and semigroups, Illinois J. Math. 7 (1963), 148-173. MR 26 \#1726.

7. —, A nonlinear integral operation, Illinois J. Math. 8 (1964), 621-638. MR $29 \# 5082$.

8. R. H. Martin, Jr., A bound for solutions to Volterra-Stieltjes integral equations, Proc. Amer. Math. Soc. 23 (1969), 506-512. MR 40 \#662.

9. - Bounds for solutions to a class of nonlinear integral equations, (submitted).

Georgia Institute of Technology, Atlanta, Georgia 30332 